SHORT COMMUNICATION

\title{
Explosive seed dispersal of the rainforest tree Tetraberlinia moreliana (Leguminosae - Caesalpinioideae) in Gabon
}

\author{
XANDER M. VAN DER BURGT
}

Department of Plant Taxonomy, Postbus 8010,6700 ED Wageningen, The Netherlands

KEY WORDS: dispersa! distance, explosive dehiscence, seed dispersal, seed trajectory.

Explosive or ballistic seed dispersal has been found in many plant species, genera and families (Ridley 1930); in both 'annuals and perennials, herbs and trees, and in open and shaded, wet and dry habitats' (Stamp \& Lucas 1983). In the Leguminosae, explosive dehiscence 'is found most frequently in herbs (...), but also occurs in high forest trees, from which large seeds lacking dispersal devices are thrown many metres away' (van der Pijl 1982: 155).

Little information is available about explosive dispersal by trees, although this dispersal method occurs frequently in specific forest types. For example, "leguminous trees with explosively dehiscent pods dominate most primary and secondary dry forest and woodland types in the Zambesian Regional Centre of Endemism' (Bingham 1994). Unpublished observations by scientists of the Department of Plant Taxonomy in Wageningen have shown that explosive dispersal is a common feature of caesalpinioid trees in the African rainforests. In this paper, some observations and experiments on the explosive dispersal of the large rainforest tree Tetraberlinia moreliana Aubréville are described. The research was carried out in Rabi, Gabon, at $1^{\circ} 55^{\prime} \mathrm{S}$ and $9^{\circ} 5 \mathrm{l}^{\prime} \mathrm{E}$, and at c. $40 \mathrm{~m}$ altitude. Rabi has an average annual rainfall of $2166 \mathrm{~mm}$ (1987-1993), and a dry season from June to September. The prevailing wind direction is SSW.

The distribution of $T$. moreliana is confined to west Gabon and a small area in south-west Cameroon (Rietkerk et al. 1995). T. moreliana can attain a height of $51 \mathrm{~m}$. The crown has the shape of a hemisphere, and can emerge above the surrounding canopy. The pods are woody, and only grow on the crown surface, beyond the outermost leaves. The longitudinal axis of a pod usually has a horizontal orientation, like a pennant, but can point in any horizontal direction 


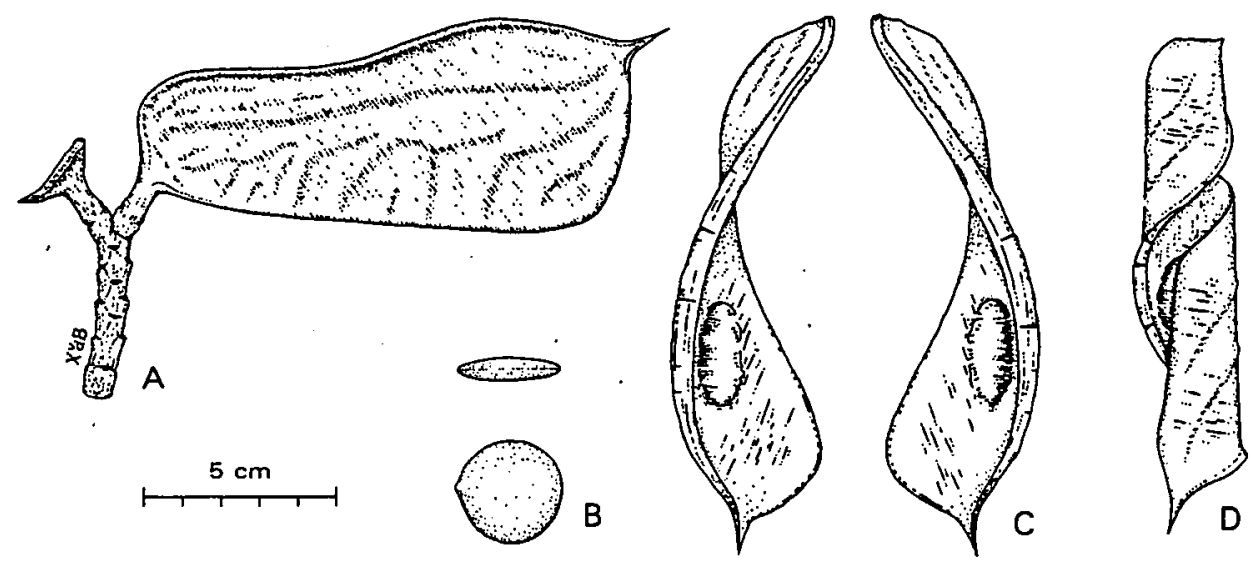

Figure 1. Pods and seeds of Tetraberlinia moreliana. A: Fruiting pedicel with pod, and stalk of exploded pod. B: Seeds have a discoid shape. C: Valves immediately after dehiscence. D: Completely dried valve.

(Figure 1A). The discoid seeds (Figure 1B) have an average weight of $2.53 \mathrm{~g}$. A pod may contain 0 to 4 seeds, with an average of 2.0 seeds. In the study area, seeds were dispersed from December 1993 to February 1994. The dehiscence of a pod is caused by tension that builds up between the two valves during drying. Mature pods explode with a crack during periods of sunshine. Observations on falling seeds indicate that a dehiscing pod can impart a large velocity to its seeds. Seeds are sometimes partly imbedded in firm ground. Some seeds are buried in soft soil at a considerable angle to the vertical.

The dispersal distance of an explosively dispersed seed is defined as the horizontal distance between this seed and the nearest edge of the crown. The positions of seeds dispersed beyond $50 \mathrm{~m}$ were determined at a tree near an artificial forest edge. The top of the tree was $48 \mathrm{~m}$ above the observation area. Seeds dispersed less than $50 \mathrm{~m}$ were not registered due to the dense vegetation and uneven topography near the tree. Dispersed seeds were collected daily, during the time when pods in the tree were exploding. Seeds did not bounce, because the area was sandy. None of the near-by trees dispersed their seeds by explosion during the study period. The total number of pods produced by the tree was estimated by counting the number of valves in four plots of $1 \mathrm{~m}^{2}$ at various distances from the stem, and the percentage of seeds dispersed beyond $50 \mathrm{~m}$ was calculated. Data on maximum dispersal distance of this tree were verified by measurements on other trees in the study area.

Maximum dispersal distances of four T. moreliana trees were 60, 52, 51 and $41 \mathrm{~m}$. Their nearest branches with pods were $39,32,30$ and $24 \mathrm{~m}$ above the observation areas. Maximum dispersal distance partly depends on tree height. A total of 41 seeds was found more than $50 \mathrm{~m}$ from the main study tree (Figure 2) over a period of $20 \mathrm{~d}$. This tree produced an estimated total number of 15,000 to 20,000 seeds. Approximately $1.5-2 \%$ of these seeds were dispersed beyond $50 \mathrm{~m}$. 


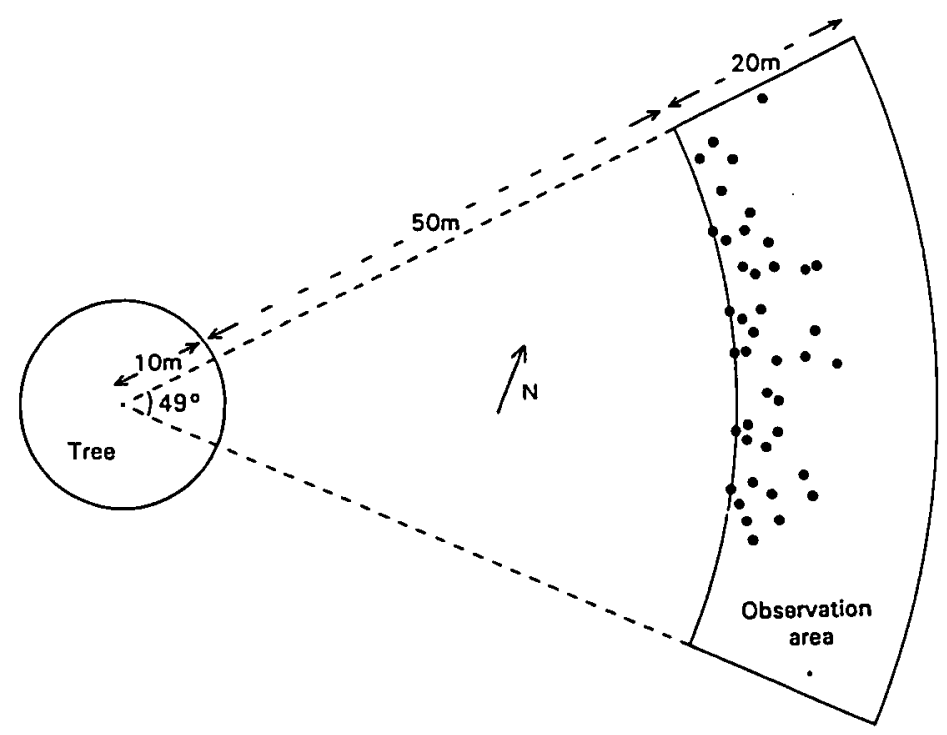

Figure 2. Positions of 41 seeds located beyond $50 \mathrm{~m}$ of the Tetraberlinia morelione study tree. The farthest dispersed seed was found $60 \mathrm{~m}$ away, horizontally measured, from the edge of the crown.

A dehiscing pod projects its seeds in two directions, approximately at right angles to the plane of the pod, but somewhat upwards, as seen from a pod with a standard orientation. The average value of this upwards angle was measured by letting detached pods explode close to the ground and in a fixed orientation. The coordinates of a seed indicate the dispersal direction in the horizontal plane only. Therefore, in order to measure the upwards angle, the detached pods were positioned upright, with their longitudinal axis in a vertical orientation. Pods were collected by climbing trees, and were fixed with their stalks in the soil of a sunny sand flat. After dehiscence of a pod, the coordinates of its dispersed seeds were established. The average upwards angle was calculated from the angles of all seeds involved.

The projection angle was determined with 11 pods that contained 18 seeds. These seeds were projected with an average upwards angle of $17.3^{\circ}(\mathrm{SD}=$ $11.0^{\circ}$ ), as seen from a pod with standard orientation. The farthest of the 18 seeds was found $23.2 \mathrm{~m}$ away from the starting point.

For any explosively dispersed seed found near a tree an optimum trajectory can be calculated, that is the trajectory with the lowest possible initial velocity necessary for the seed to reach the place where it was found. However, a seed may just as well have followed another trajectory, ending at the same spot, but with a higher initial velocity. Nevertheless, the farthest dispersed seed must have followed its optimum trajectory, because it must have been projected at the maximum velocity that a pod can give to a seed. The trajectory of the farthest dispersed seed can therefore be calculated with much more reliability than that of other seeds. 


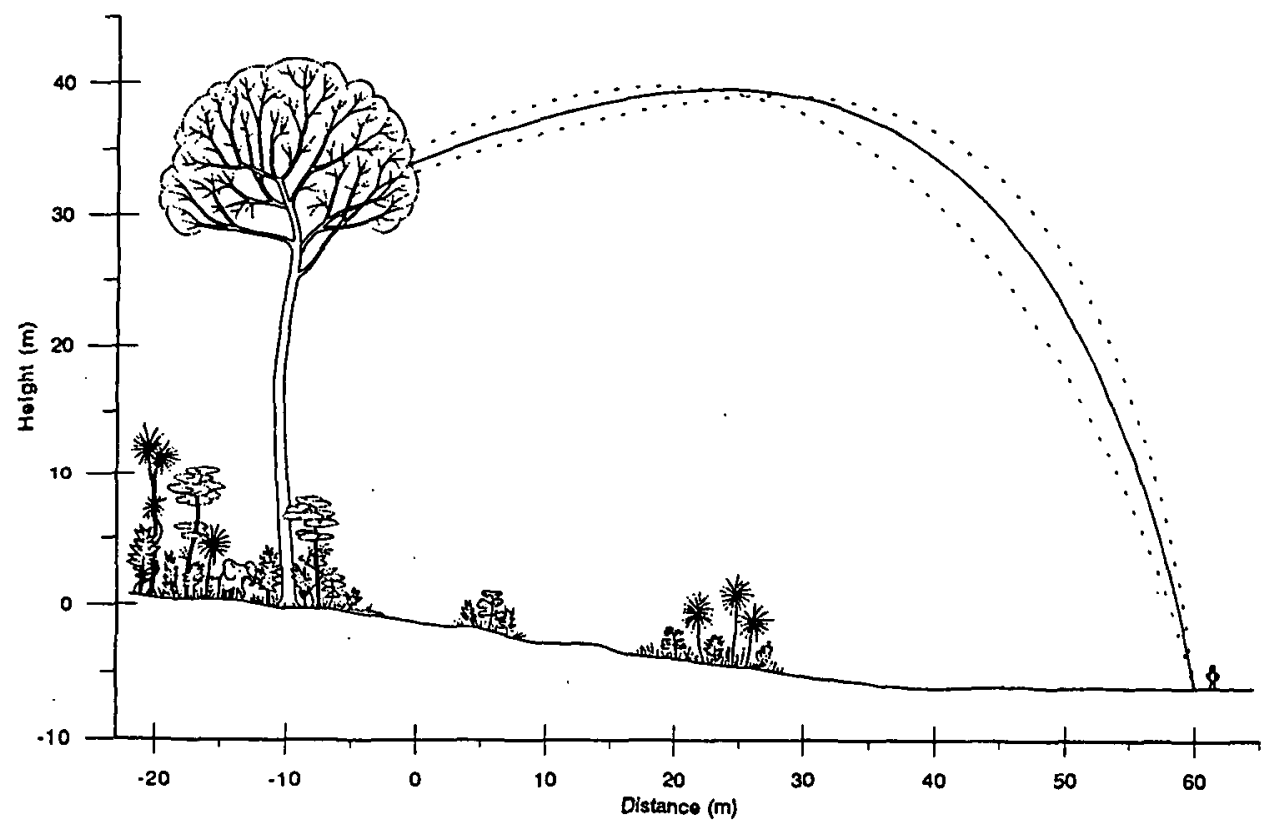

Figure 3. The optimum trajectory, at medium air resistance, of the farthest dispersed seed of a Tetraberlinia moreliana tree (solid line). Broken lines represent optimum trajectories at low and high air resistance. Seeds can pass over neighbouring tree tops before they enter the canopy.

The trajectory was derived with the assumptions that this seed: (1) started at the outside of the tree crown, (2) was not subjected to wind nor to any aerodynamic lift force, (3) was subjected to an air resistance force proportional to the square of its velocity, (4) did not move from its first point of contact with the ground. Assumption 2 is acceptable because the weather was windless during the observations. Seeds are compact and symmetric and lift forces will therefore be small. Assumption 3 is acceptable because 'it is known that for spheres with radii and velocities of practical interest' this assumption is 'a reasonable approximation to the drag force' (Parker 1977). Equations of motion, and solution methods are given by Hart \& Croft (1988) and Swaine et al. (1979). Three optimum trajectories were calculated, for low, medium and high estimates of the air resistance.

Figure 3 shows a drawing of the surroundings of the main study tree, along a transect through the stem and the place where the farthest dispersed seed was found. The optimum trajectory of this seed at medium air resistance is represented by a solid line. Its starting angle is $21.2^{\circ}$, which is in good agreement with the observed range of projecting angles. Its initial velocity is $37.1 \mathrm{~m} \mathrm{~s}^{-1}$, well below the maximum velocity of $70 \mathrm{~m} \mathrm{~s}^{-1}$ measured by Swaine \& Beer (1977) for seeds of Hura crepitans. The optimum trajectories at low and high air resistance are represented by broken lines.

The farthest dispersed $T$. moreliana seed, found at $60 \mathrm{~m}$ from the main study tree, represents the longest explosive seed dispersal distance ever 
recorded in literature. According to Hughes et al. (1994) and van der Pijl (1982) the old record was from Hura crepitans (Euphorbiaceae) and was published by Swaine \& Beer (1977). The farthest dispersed seed found by them was $41 \mathrm{~m}$ from the edge of the crown $(45 \mathrm{~m}$ from a point half-way between the stem and the edge of the crown). The longest distance so far published for a caesalpinioid tree species is $20 \mathrm{~m}$ for Eperua falcata (Forget 1989). Distances achieved by explosively dispersed seeds may be increased or reduced as a result of wind. The effect of wind on the seeds of Figure 2 was probably insignificant, because these seeds were dispersed during windless weather.

Secondary dispersal can increase the dispersal distance of explosively dispersed seeds, and can also bring seeds to a better site (Stamp \& Lucas 1983). There are many instances of secondary transport after explosion (van der Pijl 1982). Secondary dispersal of $T$. moreliana seeds by animals seems unlikely, because the seeds are not protected and will usually not survive handling by animals. The seeds lack arils, caruncles or any other apparent 'rewards' for animals. They are too smooth to attach to animals. 'Plants that maximize ballistic dispersal distance cannot exhibit structural adaptations for secondary dispersal' (Stamp \& Lucas 1983). On the other hand, seeds of $T$. moreliana are eaten by many animal species, and this may occasionally result in secondary dispersal, although this was never observed.

Seeds can pass over neighbouring tree tops before they enter the canopy (Figure 3 ), because $T$. moreliana is a tall tree, and seeds are launched slightly upwards. After entering the canopy, the trajectories of seeds are usually modified by the vegetation. Not all seeds are projected successfully. Seeds may be projected into the crown of the mother tree, into the canopy of neighbouring trees or at a low initial velocity. However, nothing is known about chances of seeds at various distances from the mother tree to become adult trees.

In the Appendix, an overview is given of caesalpinioid tree species that are indigenous to Gabon and use the explosive dispersal method. Many of these species will achieve modest distances in comparison with $T$. moreliana, because their pods are less strong, or because they are understorey trees, but some species may be able to achieve longer distances. Most species in the African genus Monopetalanthus (about 17 species) are rainforest trees of up to $59 \mathrm{~m}$ height, with powerful ballistic mechanisms.

Several advantages are associated with the dispersal of seeds some distance from the maternal plant (Howe \& Smallwood 1982). In spite of these advantages, the question is asked why T. moreliana, and many other African tree species, uses a dispersal method that seems inefficient, because of a small and strictly limited maximum dispersal distance (Figure 2), compared to many wind-, water- or animal-dispersed species. A possible answer is that, in addition to the explosive dispersal, there may exist some type of long-distance dispersal. A second answer may be that 'the evolution towards 
(...) reduced mobility appears to be an adaption enabling the species to dominate stable habitats' (Bingham 1994). A third possible answer is that 'successful dispersal may be less a function of distance (...) than of arrival in particular kinds of sites' (Willson 1993). The Appendix shows that, in Gabon, almost all Caesalpinioideae from the tribe Macrolobieae (old name: Amherstieae; Breteler 1995) are explosive dispersers, but the tribe exhibits another important ecological characteristic: 'ectomycorrhizae are a consistent feature of African Amherstieae' (Alexander 1989). 'Seedlings of (. . .) Tetraberlinia species may have to be within a critical distance of the parent tree to become infected' with ectomycorrhizae 'and so establish an advantage over competing species' (Newbery et al. 1988). More research should reveal if the correlation between explosive seed dispersal and ectomycorrhizae in Macrolobieae is causal.

\section{ACKNOWLEDGEMENTS}

The fieldwork was made possible by Shell Gabon and by C. D. A. Pauluis. Further help was given by A. M. Louis of the Cenarest in Libreville, and by F. J. Breteler, L. J. G. van der Maesen, J. J. Wieringa and J. J. F. E. de Wilde. of the Department of Plant Taxonomy in Wageningen.

\section{LITERATURE CITED}

ALEXANDER, I. J. 1989. Systematics and ecology of ectomycorrhizal legumes. Pp. $607-624$ in Stirton, C. H. \& Zarucchi, J. L. (eds). Advances in legume biology. Missouri Botanical Garden, St Louis.

AUBRÉVILLE, A. 1968. Flore du Gabon, 15, Légumineuses - Ceesalpinioidées. Muséum National d'Histoire Naturelle, Paris. 362 pp.

BINGHAM, M. G. 1994. A Zambesian success story: the explosively dehiscent pod. Pp. 1321-1329 in Seyani, J. H. \& Chikuni, A. C. (eds). Proceedings XIIIth AETFAT. National Herbarium Malawi.

BRETELER, F. J. 1995. The boundary between Amherstieae and Detarieae (Caesalpinioideae). In Crisp, M. \& Doyle, J. J. (eds). Advances in Legume Systematics 7:53-61. Royal Botanic Gardens, Kew.

FORGET, P. M. 1989. La régéneration naturelle d'une espèce autochore de la forêt Guyanaise: Eperua falcata Aublet (Caesalpiniaceae). Biotropica 21:115-125.

HART, D. \& CROFT, T. 1988. Modelling with projectiles. Ellis Horwood, Chichester. 152 pp.

HOWE, H. F. \& SMALLWOOD, J. 1982. Ecology of seed dispersal. Annual Review of Ecology and Systematics 13:201-228.

HUGHES, L., DUNLOP, M., FRENCH, K., LEISHMAN, M. R., RICE, B., RODGERSON, L., WESTOBY, M. 1994. Predicting dispersal spectra: a minimal set of hypotheses based on plant attributes. Joumal of Ecology 82:933-950.

NEWBERY, D. M., ALEXANDER, I. J., THOMAS, D. W. \& GARTLAN, J. S. 1988. Ectomycorrhizal rain-forest legumes and soil phosphorus in Korup National Park, Cameroon. New Phytologist 109:433450.

PARKER, G. W. 1977. Projectile motion with air resistance quadratic in the speed. American Joumal of Physics 45: 606-610.

RIDLEY, H. N. 1930. Dispersal of plants throughout the world. Reeve \& Co., Ashford. 744 pp.

RIETKERK, M., KETNER, P. \& WILDE, J. J. F. E. DE. 1995. Caesalpinioideae and the study of forest refuges in Gabon: preliminary results. Adansonia 17B:95-105.

STAMP, N. E. \& LUCAS, J. R. 1983. Ecological correlates of explosive seed dispersal. Oecologia 59:272278.

SWAINE, M. D. \& BEER, T. 1977. Explosive seed dispersal in Hure crepitans L. (Euphorbiaceae). New Phytologist 78:695-708

SWAINE, M. D., DAILUBA, T. \& BEER, T. 1979. On the theory of explosively dispersed seeds: a correction. New Phytologist 82:777-781. 
VAN DER PIJL, L. 1982. Principles of dispersal in higher plants. Springer, Berlin-Heidelberg-New York. $215 \mathrm{pp}$.

WILLSON, M. F. 1993. Dispersal mode, seed shadows, and colonization patterns. Vegetatio 107/108:261280.

Accepted 21 June 1996

\section{APPENDIX 1}

Explosive dispersal of caesalpinioid tree species in Gabon

A survey of caesalpinioid species in Gabon is given in Aubréville (1968). The occurrence of explosive dispersal in these species was determined by field observations and herbarium study of pods. All studied samples are preserved at the Herbarium Vadense, Wageningen. A tree species was considered to use explosive dispersal if its pods exploded, either on the tree or during drying of botanic samples, or if the dry valves of its pods have similar curved shape to valves of $T$. moreliana (Figure ID) and other species that were observed to have explosive pods.

Of the approximately 136 caesalpinioid tree species indigenous to Gabon, at least 74 use explosive seed dispersal. It is a characteristic feature for the tribe Macrolobieae (at least 55 out of 66 tree species): Anthonotha (2 of 9 species), Aphanocalyx (2), Berlinia (5), Brachystegia (2), Cryptosepalum (1 of 3), Didelotia (5), Gilbertiodendron (15), Julbernardia (3), Leonardendron (1), Librevillea (1), Microberlinia (1), Monopetalanthus (9), Oddoniodendron (2), Paraberlinia (1), Pellegriniodendron (1), Tetraberlinia (3), Toubaouate (1). Detarieae (at least 18 out of 59 tree species): Augouardia (1), Baikiaea (1 of 2), Crudia (1 of 2), Eurypetalum (2), Gilletiodendron (1 of 2), Hymenostegia (7), Loesenera (2), Neochevalierodendron (1), Plagiosiphon (1 of 3), Scorodophloeus (1). Caesalpinieae (1 out of 3 tree species): Stachyothyrsus (1). Cassieae ( 0 out of 8 tree species). Other caesalpinioid tree species may be wind, water or animal dispersers. 